Research Paper

\title{
ZHX1 Inhibits Gastric Cancer Cell Growth through Inducing Cell-Cycle Arrest and Apoptosis
}

\author{
Xingjie Ma*, Minlu Huang*, Zhenqiang Wang, Bingya Liu, Zhenggang Zhu, Chen Li ${ }^{\bowtie}$ \\ Department of Surgery, Shanghai Key Laboratory of Gastric Neoplasms, Shanghai Institute of Digestive Surgery, Ruijin Hospital, Shanghai Jiao Tong \\ University School of Medicine, Shanghai 200025, China \\ * Xingie Ma and Minlu Huang contributed equally to this work. \\ $\triangle$ Corresponding author: Chen Li, Department of Surgery, Ruijin Hospital, Shanghai Jiao Tong University School of Medicine, No. 197, Ruijin 2 Rd, \\ Shanghai, China 200025. Fax: +86-21-64373909; Phone: +86-21-64370045-611007; E-mail: leedoctor@sina.com \\ ( ) Ivyspring International Publisher. Reproduction is permitted for personal, noncommercial use, provided that the article is in whole, unmodified, and properly cited. See \\ http://ivyspring.com/terms for terms and conditions.
}

Received: 2015.06.17; Accepted: 2015.09.21; Published: 2016.01.01

\begin{abstract}
Zinc-fingers and homeoboxes $1(\mathrm{ZHX1})$ was implicated in the carcinogenesis of human cancers. However, little is known about the role of ZHXI in gastric cancer (GC). Here, we found that $\mathrm{ZHXI}$ was downregulated in GC tissues compared with paired non-tumor tissues and loss of ZHX1 expression correlated with aggressive clinical characteristics of GC patients. ZHX1 induced $\mathrm{Gl} / \mathrm{S}$ arrest by decreasing cyclin $\mathrm{Dl}$ and cyclin $\mathrm{E}$ expression, and induced cell apoptosis by $\mathrm{Bcl} 2$ downregulation and Bax and cleaved Caspase- 3 upregulation. Our findings revealed that ZHXI could inhibit cell growth through inducing cell-cycle arrest and apoptosis in GC.
\end{abstract}

Key words: ZHX1; Gastric cancer; Cell growth; Cell cycle; Apoptosis

\section{Introduction}

Gastric cancer (GC) is one of the most common malignancies and a major health burden worldwide, with an estimated 951,600 new cases and 723,100 related deaths in 2012 [1]. Despite great improvements in diagnosis and treatment, the prognosis of patients with advanced GC is still generally poor [2]. The carcinogenesis of GC is a complex multistep procession mediated by epigenetic and genetic alterations of oncogenes and tumor suppressor genes, but the underlying molecular mechanisms are still elusive. Furthermore, the currently available biomarkers fail to effectively predict early-stage GC. Therefore, a better understanding of the molecular processes that drive GC could help identify sensitive and specific biomarkers to predict GC in early stage.

Zinc-fingers and homeoboxes protein 1 (ZHX1) is a member of the small ZHX family, which also includes ZHX2 and ZHX3 [3-5]. The ZHX family members contain two $\mathrm{C}_{2} \mathrm{H}_{2}$ zinc-finger ( $\mathrm{Znf}$ ) motifs and four to five homeodomains, and belong to the Znf class of homeodomain transcription factors. Recently, the role of ZHX family in human cancers has been the subject of much attention. ZHX2 was reported to be a tumor suppressor in hepatocellular carcinoma (HCC) $[6,7]$ and Hodgkin lymphoma [8, 9], and low expression of ZHX2 predicted poor prognosis in multiple myeloma [10]. ZHX1 was generally downregulated in HCC tissues and overexpression of ZHX1 could inhibit the growth of SMMC-7721 cells [11]. These studies suggested that the ZHX family may be involved in the carcinogenesis of human cancers.

ZHX1 consists of 873 amino acid residues and contains a nuclear localization signal (NLS) at the C-terminal [12]. ZHX1 can form homodimers or heterodimers with NF-YA [13], BS69 [14], and DNMT3B [15]. ZHX1 functions as a novel transcription repressor; however, its downstream target genes and the DNA-binding sequence have not yet been identified. We previously reported that miR-199a-3p can promote the progression of GC by directly targeting ZHX1 [16]. However, little is known about the exact role of ZHX1 in GC. 
In this study, we investigated the expression pattern of ZHX1 in GC tissues by immunohistochemistry and its relationship to clinicopathological features. In addition, we further examined the biological function of ZHX1 in GC cell lines.

\section{Materials and Methods}

\section{Cell culture and tissue specimens}

The human gastric cancer cell lines BGC-823, SGC-7901, NCI-N87, MKN-45, MKN-28, and AGS were obtained from Shanghai Institutes for Biological Sciences, Chinese Academy of Sciences. HS-746T cells were purchased from American Type Culture Collection (Manassas, VA, USA). The cells were cultured in RPMI 1640 supplemented with $10 \%$ fetal bovine serum (FBS) at $37^{\circ} \mathrm{C}$ in a humidified atmosphere with $5 \% \mathrm{CO}_{2}$.

GC tissues and paired non-tumor tissues were obtained from 112 patients undergoing radical gastrectomy at Ruijin Hospital, Shanghai Jiao Tong University School of Medicine between December 2011 and July 2013. None of the patients received chemotherapy or radiotherapy prior to surgery. All tissues were embedded into a tissue microarray. This study was approved by the Ethics Committee of Ruijin Hospital and all patients provided written informed consent.

\section{Immunohistochemistry and scoring}

Immnunohistochemistry staining was performed according to the previously described protocol [17]. We used a rabbit polyclonal ZHX1 antibody (1:100; Abcam, USA). The tissue sections were independently evaluated and scored by two pathologists who were blinded to the clinical information of the subjects. Nuclear staining of cells was considered to be positive immunostaining. The intensity of ZHX1 nuclear staining was graded as 0 (no staining), 1 (weak staining), 2 (moderate staining), or 3 (high staining). The proportion of positive cells was scored as follows: $0(0 \%), 1(1-25 \%), 2(26-50 \%), 3(51-75 \%)$, or $4(76-100 \%)$. The final immunohistochemistry staining score of each tissue sample was calculated by multiplying the staining intensity by proportion of positive cells; the total expression of ZHX1 was determined as negative expression (0-3) or positive expression (4-12).

\section{Establishment of stable cell lines}

The pEGFP-ZHX1 plasmid, which expresses ZHX1-GFP fusion protein, was kindly provided by Prof. Kazuyu Yamada (Matsumoto University Graduate School of Health Science). The pEGFP-C1 negative control plasmid was purchased from GenePharm (Shanghai, China). We transfected pEGFP-ZHX1 or
pEGFP-C1 plasmids into NCI-N87 cells using Lipofectamine 2000 (Invitrogen, Carlsbad, CA, USA) according to the manufacturer's instructions. Stable clones were selected by continuous treatment with $1000 \mu \mathrm{g} / \mathrm{mL}$ G418 for 4 weeks.

The shRNA sequence designed to target ZHX1 was 5'-TCAGACCATAACTGTTATT-3' and the negative control sequence was 5'-TCTCCGAACGT GTCACGT-3'. The oligoduplexes were cloned into the GV248 vector (Genechem, Shanghai, China). The shRNAs were transfected into SGC-7901 cells using Lipofectamine 2000. Stable transfectants were selected by culturing with $2 \mu \mathrm{g} / \mathrm{mL}$ puromycin for 3 weeks.

\section{Real-time RT-PCR and western blot}

Total cell RNAs were extracted using Trizol reagent (Invitrogen, Carlsbad, USA) according to the manufacturer's instructions and the concentration was measured by a spectrophotometer. The Reverse Transcription kit (Promega, Madison, WI, USA) was used to synthesize cDNA. The mRNA level of ZHX1 (sense: 5'-CAGGTCAGAGAGTGGTTTGC-3'; anti-sense: 5'-GTCGTGGAGGTTCCCAAGT-3') was determined by quantitative real-time RT-PCR (qRT-PCR) using SYBR Green PCR master mix (Applied Biosystems, Foster City, USA). The relative expression of ZHX1 was normalized with GAPDH (sense: 5'-CCCATCACCATCTTCCAGGAG-3'; anti-sense: 5'-CTTCTCCATGGTGGTGAAGACG-3') and was calculated with the $2^{-\Delta \mathrm{CT}}$ method.

Total proteins from cells were extracted using RIPA buffer (Solarbio, Beijing, China) and the protein concentration was measured by BCA Protein Assay Kit (Pierce, Rockford, USA). Western blot was performed according to the standard protocol, with primary antibodies against ZHX1 (1:2000, Abcam), cyclin D1 (1:1000, Cell Signaling Technology (CST), USA), cyclin E (1:1000, CST, USA), Bax (1:1000, CST, USA), Bcl2 (1:1000, CST, USA) and cleaved Caspase-3 (1:1000, CST, USA). GAPDH was used as a control to confirm equal loading of proteins. Tannon $2500 \mathrm{im}-$ aging system was used to analyze the band intensities.

\section{Cell proliferation assay}

Cell proliferation was evaluated by Cell Counting Kit-8 assay (Dojindo, Kumamoto, Japan) according to the manufacturer's instructions. Briefly, cells were seeded into 96-well plates at $1.5 \times 10^{3}$ per well and incubated for $0 \mathrm{~h}, 24 \mathrm{~h}, 48 \mathrm{~h}, 72 \mathrm{~h}$, and $96 \mathrm{~h}$. Then, $10 \mu \mathrm{L}$ of CCK-8 solution was added into the wells and after $3 \mathrm{~h}$, the plates were examined using a spectrophotometer. Five replicate wells were tested per assay, and all experiments were performed in triplicate. 


\section{Soft agar colony formation assay}

Cells $\left(1 \times 10^{3}\right.$ per well) were resuspended in $0.3 \%$ soft agar in RPMI 1640 containing 20\% FBS and layered on top of $0.6 \%$ solidified agar in RPMI 1640 containing 20\% FBS in six-well plates. After 3 weeks incubation, colonies containing at least 50 cells were counted and photographed.

\section{Cell cycle and apoptosis analysis}

For cell-cycle analysis, cells were fixed in $75 \%$ cold ethanol and stored at $4^{\circ} \mathrm{C}$ overnight. The next day, the fixed cells were washed with phosphate-buffered saline twice, treated with RNase A, and stained with propidium iodide (PI) for $30 \mathrm{~min}$ at room temperature in the dark. Cell-cycle distribution was analyzed by flow cytometry (FACSCalibur, Becton Dickinson, MD, USA).

Apoptotic cells were detected using the Annexin V-FITC Apoptosis Detection kit (BD Pharmingen) according to the manufacturer's instructions. Briefly, the cells $\left(3 \times 10^{5}\right)$ were harvested and stained with $3 \mu \mathrm{L}$ Annexin V-FITC and $5 \mu \mathrm{L}$ PI for $15 \mathrm{~min}$ in the dark. Cells were immediately analyzed by flow cytometry.

\section{Cell migration assays}

For cell migration assay, $200 \mu \mathrm{L}$ serum-free RPMI-1640 containing $1 \times 10^{5}$ cells was added into the upper chamber of 8-um pore transwells (Corning, Lowell, MA, USA). The lower chamber contained 600 $\mu \mathrm{L}$ RPMI-1640 with $10 \%$ FBS as a chemoattractant. The chambers were incubated for another $24 \mathrm{~h}$ at $37^{\circ} \mathrm{C}$, and then cells were fixed by $10 \%$ formalin for 30 min and stained with $0.5 \%$ crystal violet for $15 \mathrm{~min}$. Finally, cells attached to the filter were quantified by inverted microscopy.

\section{In vivo experiments}

Stable transfectants $\left(2 \times 10^{6}\right.$ in $100 \mu \mathrm{L}$ of sterilized phosphate-buffered saline) were injected subcutaneously into the dorsal flank of 4-week-old male BALB/c nude mice (five mice per group). Tumor nodules were measured every week with a caliper and the tumor volumes were calculated using the following formula: $V=$ tumor length $\times$ tumor width $2 / 2$. After 35 days, all mice were sacrificed and the tumors were excised, weighed, embedded in paraffin, and examined histologically. Mice received humane care and the experimental protocols were approved by the Institutional Animal Care and Use Committee of the Shanghai Jiao Tong University.

\section{Statistical analysis}

All statistical analyses were performed by IBM SPSS Statistical software (version 18.0). Correlation between ZHX1 nuclear staining and clinicopatholog- ical features was analyzed using Pearson's Chi-square $(\chi)$ test. Student's $t$ test or one-way analysis of variance was used for statistical analysis when appropriate. A two-tailed value of $P<0.05$ was considered to be statistically significant.

\section{Results}

\section{Expression and distribution of $\mathrm{ZHX} \mathbf{1}$ in GC tissues}

We analyzed the expression and distribution of ZHX1 protein in 112 GC tissues and paired non-tumor tissues by immunohistochemistry (Figure 1). Staining of ZHX1 was predominantly observed in the nuclei both in GC tissues and paired non-tumor tissues. Statistical analysis revealed that nuclear staining of ZHX1 was significantly lower in GC tissues compared with non-tumor tissues. Among the 112 patients, positive nuclear expression of ZHX1 was detected in 72/112 (64.3\%) non-tumor tissues and 43/112 (38.4\%) tumor tissues. The differential expression of ZHX1 in GC tissues and non-tumor tissues had significant statistical significance $(P<0.001$, Table 1$)$.

Table 1. The frequency of nuclear ZHXI expression in GC tissues and paired non-tumor tissues

\begin{tabular}{lllll}
\hline Classification & Total & ZHX1 (-) & ZHX1 (+) & P value \\
\hline Tumor tissue & 112 & $69(61.6 \%)$ & $43(38.4 \%)$ & $<0.001$ \\
No-tumor tissue & 112 & $40(35.7 \%)$ & $72(64.3 \%)$ & \\
\hline
\end{tabular}

We further investigated the correlation between the expression of nuclear ZHX1 in GC tissues and clinicopathological features using Pearson's Chi-square $(\chi)$ test. As shown in Table 2, loss of nuclear ZHX1 expression was significantly correlated with large tumor size $(P=0.037)$, poor differentiation $(P=0.023)$, advanced TNM stage $(P=0.008)$, and deep invasion depth $(P=0.016)$, but not correlated with age $(P=0.722)$, sex $(P=0.449)$, location $(P=0.612)$, and lymph node metastasis $(P=0.269)$. Thus, these findings suggest that loss of ZHX1 is likely to be involved in the carcinogenesis of GC.

\section{Establishment of sable transfectants and lo- calization of transfected $\mathrm{ZHX} \mathbf{1}$}

The protein and mRNA expression levels of ZHX1 were evaluated in seven GC cell lines by western blot and qRT-PCR (Figure 2A). Among the seven GC cell lines, NCI-N87 and SGC-7901 cell lines displayed the lowest and highest endogenous ZHX1 levels, respectively. Thus, we chose NCI-N87 cells for overexpression experiments and SGC-7901 cells for knockdown experiments. We established a stable ZHX1 upregulated NCI-N87 cell clone and a ZHX1 
downregulated SGC-7901 cell clone. Changes in ZHX1 levels were confirmed by qRT-PCR and western blotting analysis (Figure 2B).

We next performed fluorescence microscopy to evaluate the subcellular localization of ectopically expressed ZHX1 protein in NCI-N87 cells. Transfection of cells with a plasmid encoding a ZHX1-GFP fusion protein revealed that green fluorescent signal was detected only in the nuclei. In comparison, green fluorescence was observed throughout the cells transfected with the pEGFP-C1 plasmid that only expresses GFP protein (Figure 2C). These findings confirmed that ZHX1 protein is localized in the nuclei.

\section{ZHXI inhibits proliferation in GC cell lines}

CCK-8 assay showed that ectopic expression of ZHX1 in NCI-N87 cells significantly suppressed the cell growth compared with the control cells (Figure $3 \mathrm{~A} ; P<0.05)$. In contrast, reducing ZHX1 levels in SGC-7901 cells by shRNA increased cell growth compared with control cells (Figure 3B; $P<0.05$ ). Moreover, the number of colonies of NCI-N87 cells transfected with ZHX1 was significantly lower than that of the control cells (Figure 3C; $P<0.05$ ). SGC-7901 cells transfected with shRNA formed more colonies compared with control cells (Figure 3D; $P<0.05$ ). Taken together, these findings demonstrated that ZHX1 significantly inhibits GC cell proliferation.
Table 2. Correlation of $\mathrm{ZHX} 1$ nuclear expression with clinicopathologic features

\begin{tabular}{|c|c|c|c|c|}
\hline \multirow[t]{2}{*}{ Classification } & \multirow[t]{2}{*}{ Number(n=112) } & \multicolumn{2}{|c|}{ ZHX1 nuclear expression } & \multirow[t]{2}{*}{ P-value } \\
\hline & & Negative & Positive & \\
\hline \multicolumn{5}{|l|}{ Age(years) } \\
\hline$<65$ & 44 & 28 & 16 & \multirow[t]{2}{*}{0.722} \\
\hline$\geq 65$ & 68 & 41 & 27 & \\
\hline \multicolumn{5}{|l|}{ Gender } \\
\hline Male & 76 & 45 & 31 & \multirow[t]{2}{*}{0.449} \\
\hline female & 36 & 24 & 12 & \\
\hline \multicolumn{5}{|l|}{ Differentiation } \\
\hline Well to moderate & 45 & 22 & 23 & \multirow[t]{2}{*}{0.023} \\
\hline Poor & 67 & 47 & 20 & \\
\hline \multicolumn{5}{|l|}{ Tumor size } \\
\hline$\leq 5 \mathrm{~cm}$ & 59 & 31 & 28 & \multirow[t]{2}{*}{0.037} \\
\hline$>5 \mathrm{~cm}$ & 53 & 38 & 15 & \\
\hline \multicolumn{5}{|l|}{ Location } \\
\hline Distal & 71 & 45 & 26 & \multirow[t]{2}{*}{0.612} \\
\hline Middle and proximal & 41 & 24 & 17 & \\
\hline \multicolumn{5}{|l|}{ Invasion depth } \\
\hline $\mathrm{T} 1, \mathrm{~T} 2$ & 30 & 13 & 17 & \multirow[t]{2}{*}{0.016} \\
\hline $\mathrm{T} 3, \mathrm{~T} 4$ & 82 & 56 & 26 & \\
\hline \multicolumn{5}{|l|}{ Lymph node metastasis } \\
\hline Yes & 82 & 48 & 34 & \multirow[t]{2}{*}{0.269} \\
\hline No & 30 & 21 & 9 & \\
\hline \multicolumn{5}{|l|}{ TNM stage } \\
\hline I , II & 31 & 13 & 18 & \multirow[t]{2}{*}{0.008} \\
\hline III, IV & 81 & 56 & 25 & \\
\hline
\end{tabular}
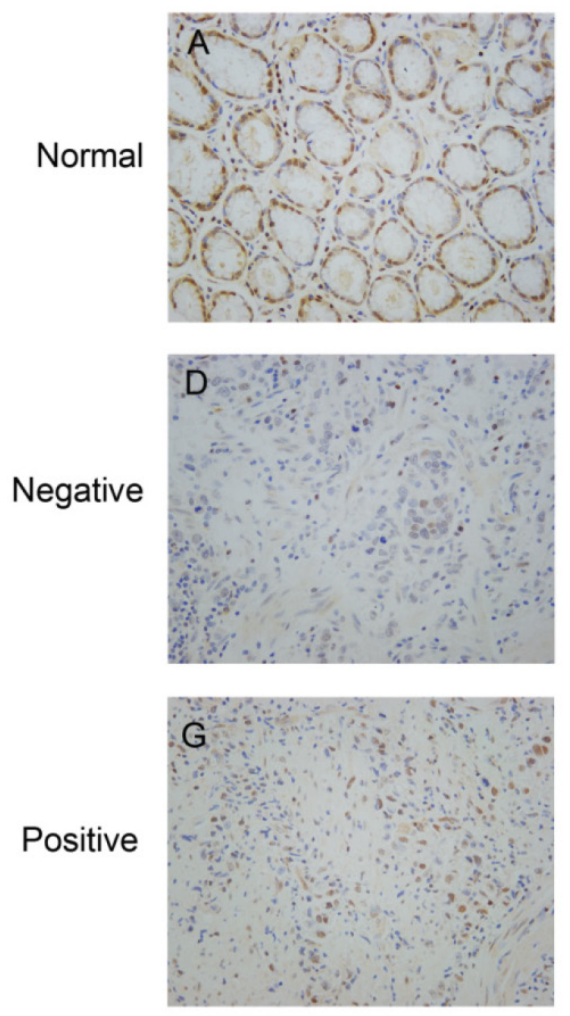
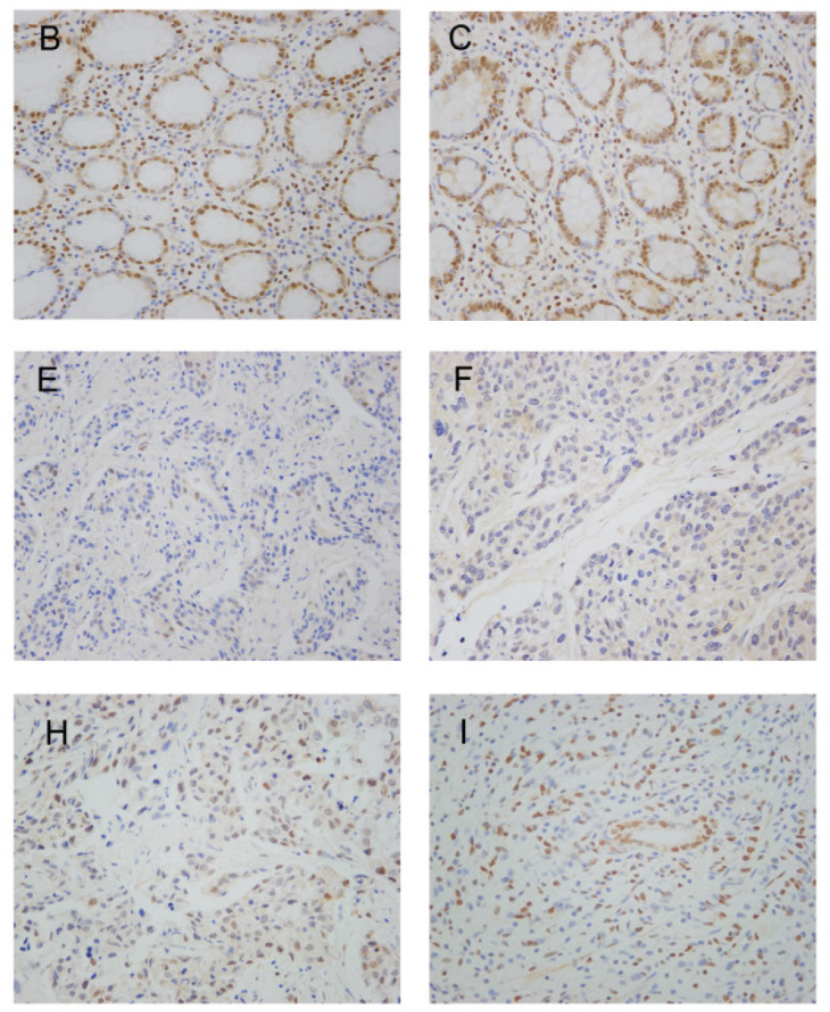

Figure 1. Representative immunohistochemistry staining of ZHXI in clinical specimens. (A, B, C) Positive ZHXI expression in non-tumor tissues (200x). (D, E, F) Negative ZHX1 expression in GC tissues (200×). (G, H, I) Positive ZHX1 expression in GC tissues (200×). 
A

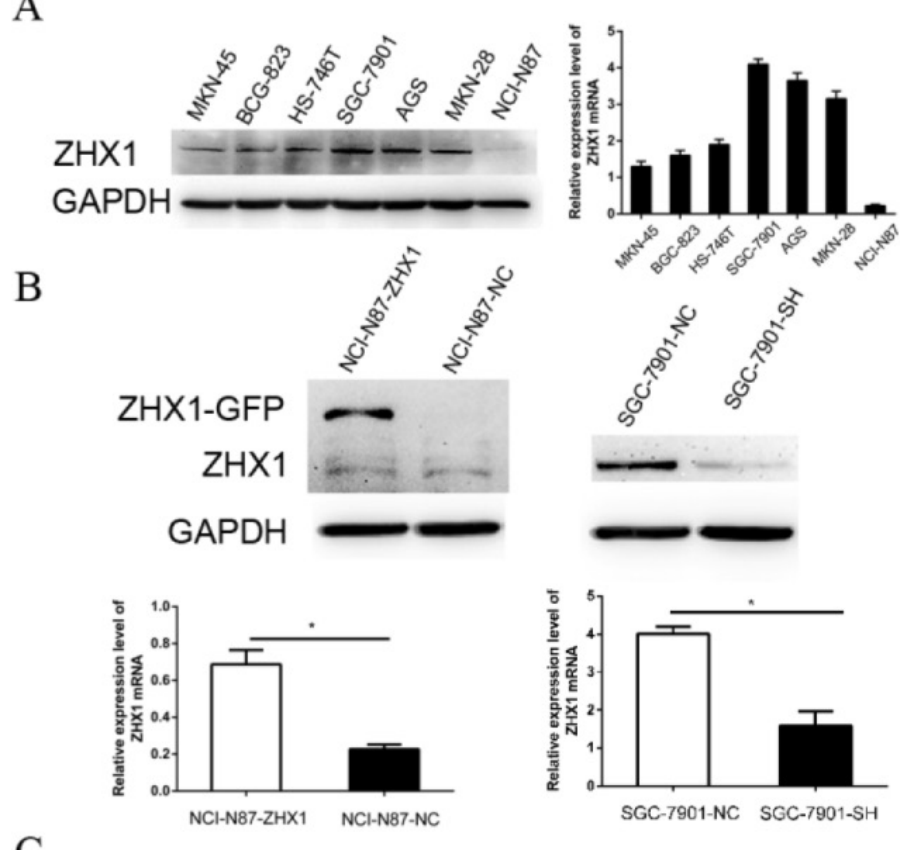

$\mathrm{C}$
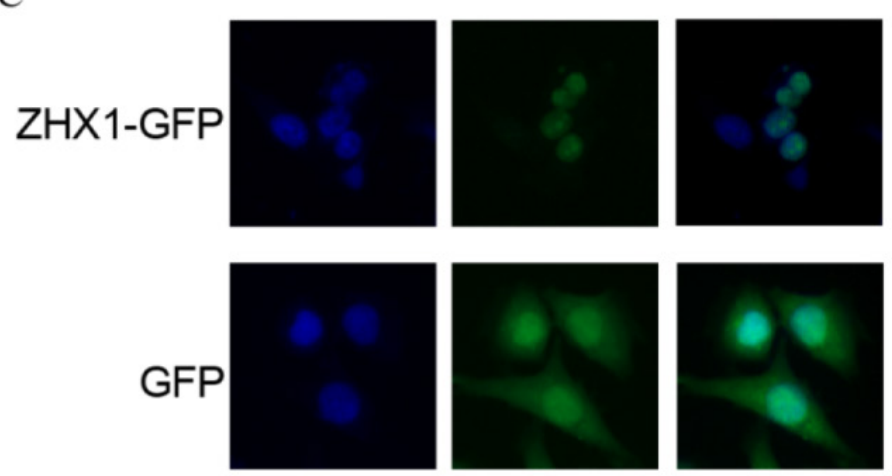

Figure 2. Expression and localization of $Z H X 1$ in $G C$ cells. (A) ZHX1 protein levels were detected by western blotting in seven $G C$ cell lines (left). Expression of ZHX1 mRNA was examined by real-time PCR in seven GC cell lines (right). (B) Stable overexpression and reduction of ZHX1 in GC cells was confirmed by western blotting and qRT-PCR (*P $<0.05$ ). (C) Subcellular distribution of ZHX1 was detected by fluorescence microscopy. The top row reveals distribution of the ZHX1-GFP fusion protein only in the nuclei; the bottom row shows distribution of GFP throughout the entire cell.

\section{ZHX1 induces apoptosis and cell-cycle arrest at G1/S phase}

The noticeable inhibition of cell proliferation by ZHX1 prompted us to assess the effects of ZHX1 on apoptosis and cell-cycle distribution of GC cells. As shown in Figure 4A, upregulation of ZHX1 level in NCI-N87 cells increased the percentage of cells in G1/G0 phase compared with controls $(62.1 \% \pm 1.9 \%$ vs. $42.2 \% \pm 2.6 \%$, respectively; $P<0.05)$ and decreased the percentage of cells in $S$ phase $(12.6 \% \pm 2.5 \%$, vs. $34.2 \% \pm 2.9 \%$, respectively; $P<0.05)$. Consistent with these results, reduction of ZHX1 level in SGC-7901 decreased the percentage of cells in G1/G0 $(55.9 \% \pm$ $1.6 \%$ vs. $64.8 \% \pm 2.6 \%$, respectively; $P<0.05$, Figure $4 \mathrm{~B})$ and increased the percentage of cells in $S$ phase $(26.7 \% \pm 1.5 \%$ vs. $19.2 \% \pm 1.7 \%$, respectively; $P<0.05$; Figure $4 \mathrm{~B})$. We evaluated the expression of several cell-cycle regulators by western blot, and the results showed that ectopic expression of ZHX1 in NCI-N87 cells reduced the expression of cyclin D1 and cyclin E, while reduction of the expression of ZHX1 in SGC-7901 cells increased the level of cyclin D1 and cyclin E (Figure 4E).

We next examined the role of ZHX1 in apoptosis by flow cytometry. The results showed that NCI-N87 cells transfected with ZHX1 showed an increased apoptosis rate compared with control cells $(7.0 \% \pm$ $2.1 \%$ vs. $32 \% \pm 3.6 \%$, respectively; $\mathrm{P}<0.05$, Figure $4 \mathrm{C}$ ), while downregulation of ZHX1 in SGC-7901 cells resulted in a reduced apoptosis rate compared with control cells $(9.6 \% \pm 1.5 \%$ vs. $16.2 \% \pm 1.1 \%$, respectively; $P<0.05$; Figure $4 \mathrm{D}$ ). We also examined the expression of apoptosis regulators and found that ectopic expression of ZHX1 in NCI-N87 cells resulted in increased expression of apoptotic proteins Bax and cleaved Caspase- 3 and decreased expression of the anti-apoptotic protein Bcl2. Downregulation of ZHX1 expression in SGC-7901 cells had the opposite effects (Figure 4F). 

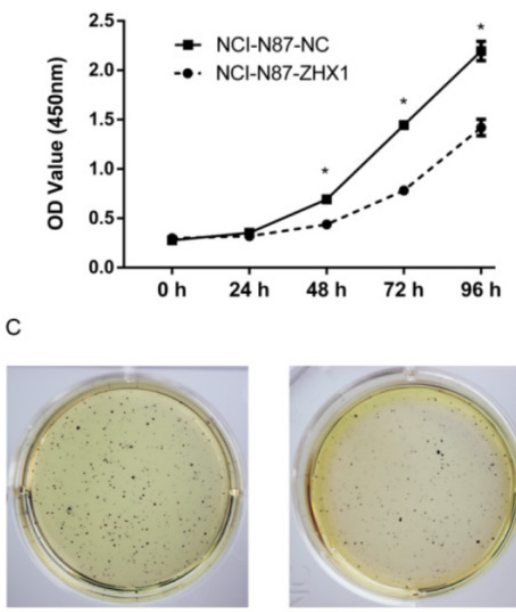

$\mathrm{NCl}-\mathrm{N} 87-\mathrm{NC}$

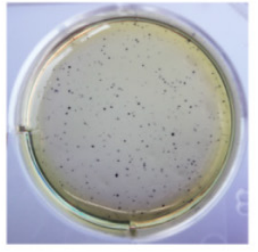

SGC-7901-NC

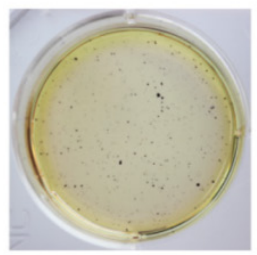

NCI-N87-ZHX1

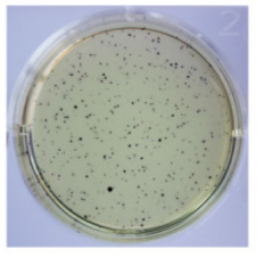

SGC-7901-SH
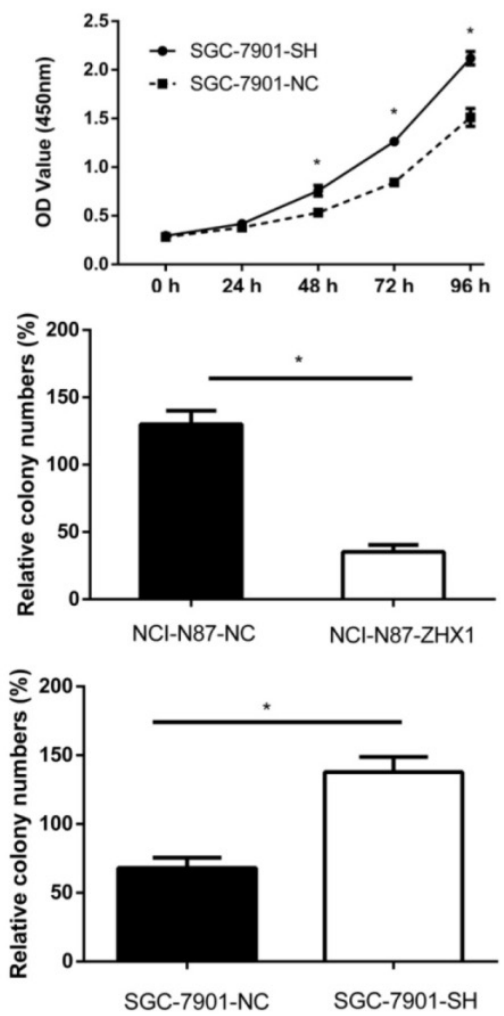

Figure 3. Ectopic expression of ZHX1 inhibits cell growth of GC in vivo. (A) Cell proliferation curves were measured by Cell Counting Kit-8 assay. The results are means of three independent experiments \pm S.D. $\left({ }^{*} P<0.05\right)$. (B) Colony formation assay results are shown on the left; the statistical results are shown on the right $(* P<0.05)$.

\section{$\mathrm{ZHX} 1$ reduces the migration capacity of $\mathrm{GC}$ cells}

Migration assays were used to examine the effect of ZHX1 on GC cell motility. As shown in Figure 5, the number of migrating cells in the NCI-N87-ZHX1 group was lower than the NCI-N87-NC group (83.3 \pm 7.6 vs. $24.7 \pm 4.5$, respectively; $P<0.05$, Figure $5 \mathrm{~A}$ ), while the SGC-7901-SH group showed increased migration compared with the control group (306.7 \pm 20.8 vs. $163.3 \pm 15.3 ; P<0.05$, Figure 5B).

\section{ZHX1 inhibits tumorigenicity in vivo}

We further examined whether ZHX1 can inhibit tumorigenicity in vivo. The stable transfectants were injected subcutaneously into the dorsal flanks of nude mice. All nude mice developed tumor nodules at the injected site, and tumor formation was monitored and analyzed by growth curves (Figure 6A, B, $P<0.05$ ). The results indicated that ectopic expression of ZHX1 could significantly suppress the tumor growth in vivo. Thirty-five days later, the mice were euthanized and the xenograft tumors were photographed (Figure 6C and 6D).

Immunohistochemistry analysis of Ki-67 antigen revealed that the percentage of $\mathrm{Ki}-67$ antigen-positive cells was remarkably reduced in the tumors from mice implanted with NCI-N87-ZHX1 group cells than those with NCI-N87-NC cells (Figure 6E; $P<0.05$ ). The percentage of Ki-67 antigen-positive cells in tumors from mice implanted with SGC-7901 cells was higher than the control group (Figure 6F; $P<0.05$ ). These data suggested that the reduced tumor growth in mice may be partly from the lower proliferation induced by $\mathrm{ZHX1}$ expression.

\section{Discussion}

$\mathrm{ZHX1}$ is a novel transcription repressor localized in the nucleus and is implicated in tumor progression, but its exact role in GC remains elusive. In this study, we examined, for the first time, the expression pattern of ZHX1 in GC tissues and its correlation with clinicopathologic features. We observed that $\mathrm{ZHX} 1$ is predominantly localized in the nuclei both in tumor cells and normal cells, and that the expression of nuclear $\mathrm{ZHX1}$ in GC tissues is lower than paired non-tumor tissues. The loss of ZHX1 protein in GC tissues may be partly because of the high expression of miR-199a-3p, which can suppress the expression of ZHX1 at a post-transcriptional level [16]. Furthermore, loss of expression of nuclear ZHX1 was correlated with large tumor size, poor differentiation, deep invasion depth, and advanced TMN stage. These findings suggested loss of expression of nuclear ZHX1 may contribute to the carcinogenesis of GC. 
A
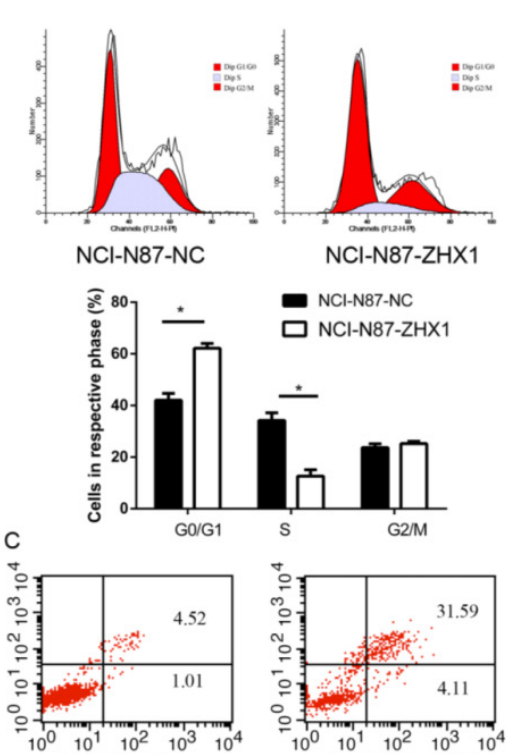

$\mathrm{NCl}-\mathrm{N} 87-\mathrm{NC}$
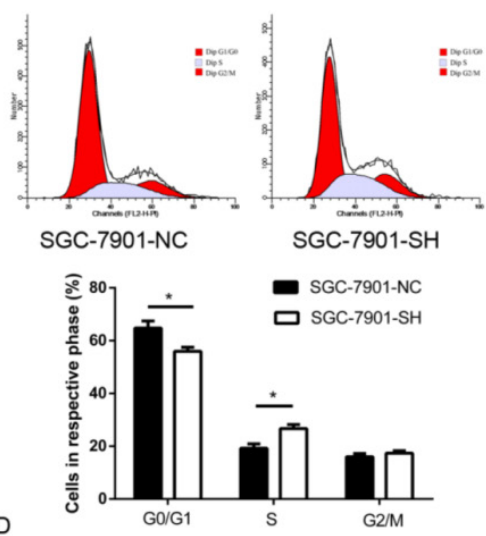

D
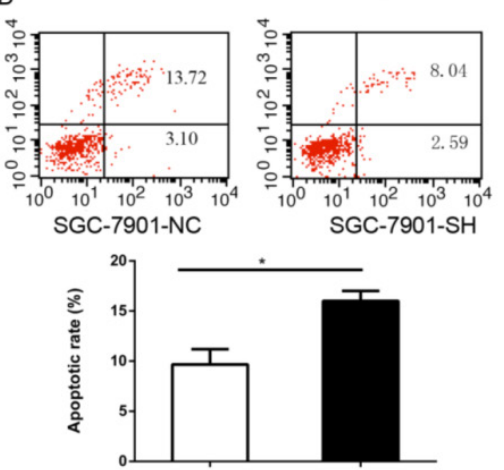

SGC-7901-SH SGC-7901-NC

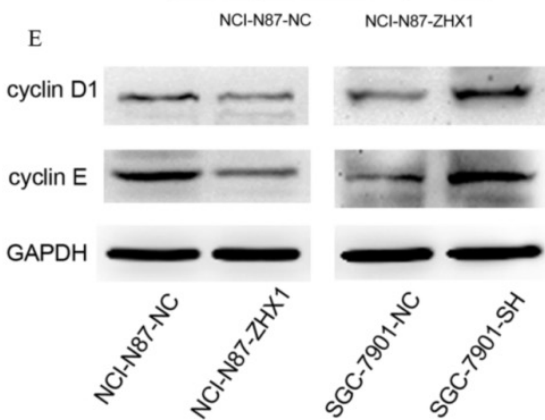

F

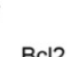

$\mathrm{Bcl} 2$

Bax

cleaved-

caspase-3

GAPDH

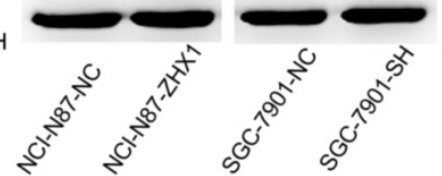

Figure 4. Effect of ZHX1 on the cell cycle and apoptosis in GC cells. (A) Ectopic expression of ZHX1 in NCl-N87 cells led to an increase of cells at G0/G1 phase and a decrease of cells at $S$ phase; the statistical results are shown below (*P<0.05). (B) Reduction of the ZHX1 level in SGC-7901 cells decreased cells at G0/G1 phase and increased cells at $S$ phase; the statistical results are shown below $(* P<0.05)$. (C) Upregulation of ZHX1 induces apoptosis in $\mathrm{NCl}-\mathrm{N} 87$ cells, and $(\mathrm{D})$ downregulation of $Z \mathrm{HX} 1$ reduces apoptosis in SGC-7901 cells. The statistical results are shown below each panel $(* P<0.05)$. (E) Western blot analysis of cell-cycle regulators cyclin $D 1$ and cyclin $E(F)$ Western bolt analysis was performed to determine the expression of $\mathrm{Bc} / 2, \mathrm{Bax}$ and cleaved caspase-3

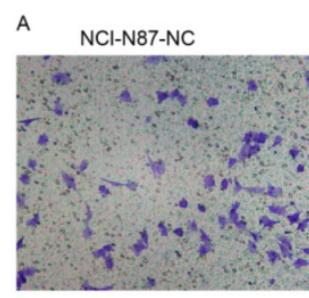

B SGC-7901-NC

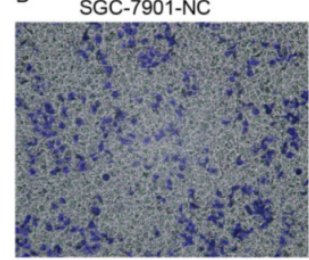

NCI-N87-ZHX1

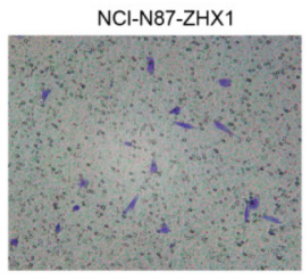

SGC-7901-SH

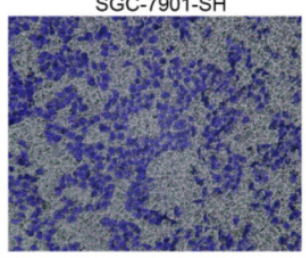

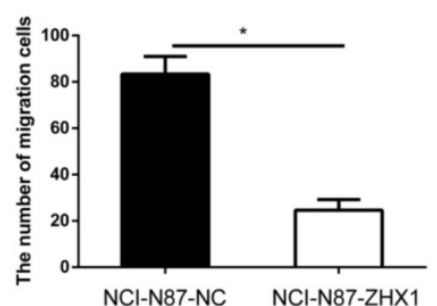

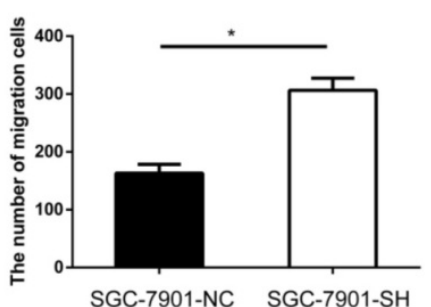

Figure 5. Effect of ZHX1 on migratory ability in GC cells. (A) Ectopic expression of ZHX1 in NCl-N87 cells inhibited cell migration; the statistical results are shown on the right $(* P<0.05)$. (B) Reduction in the ZHX1 level in SGC-7901 cells enhanced cell migration; the statistical results are shown on the right $(* P<0.05)$. 
A
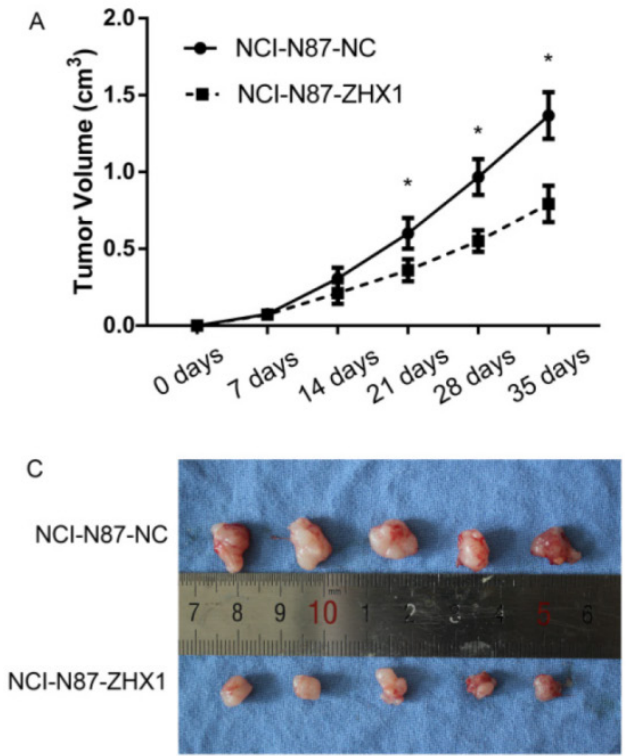

B

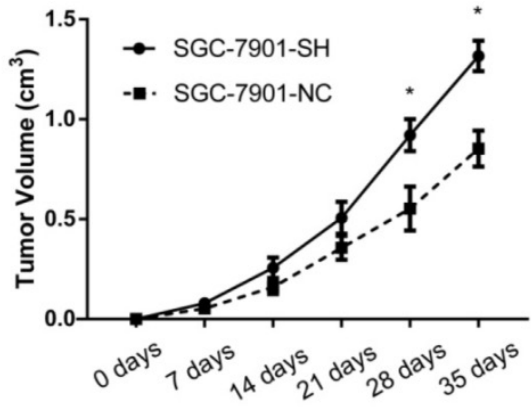

D

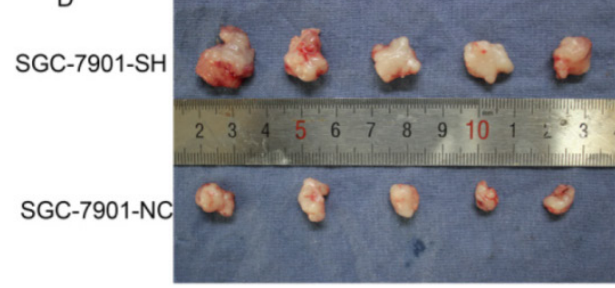

E

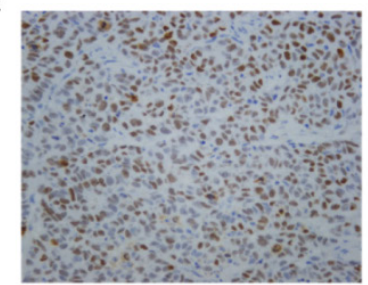

$\mathrm{NCl}-\mathrm{N} 87-\mathrm{NC}$

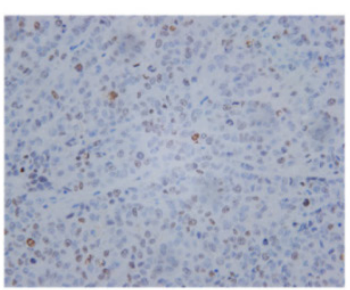

SGC-7901-NC

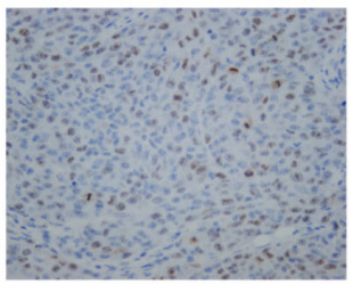

NCI-N87-ZHX1

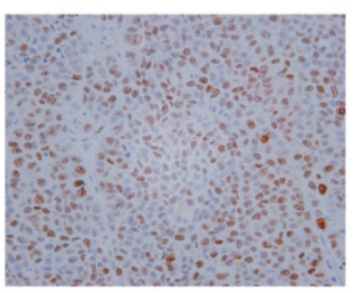

SGC-7901-SH

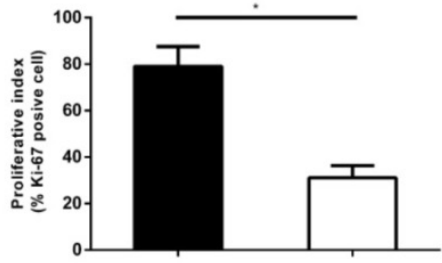

NCI-N87-NC

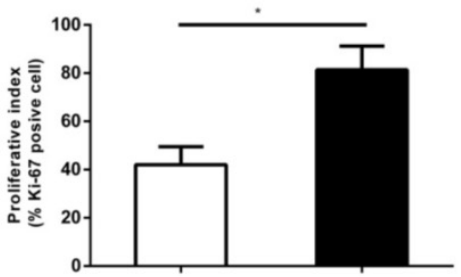

SGC-7901-NC

SGC-7901-SH

Figure 6. Effect of $\mathrm{ZHX1}$ on tumorigenic potential of $\mathrm{GC}$ cell lines in vivo. (A, B) Growth kinetics curves of tumors in nude mice implanted with $\mathrm{NCl}-\mathrm{N} 87$ cells overexpressing ZHX1 (left) or SGC-7901 cells downregulated for ZHX1 (right), compared with the indicated controls. (C, D) Photographs of tumors from NCI-N87-ZHX1/NC groups and SGC-7901-SH/NC groups. (E) Representative photographs of immunohistochemical analysis of the Ki-67 antigen in tumors from NCl-N87-ZHX1/NC groups; comparison of proliferation index is shown on the right $(* \mathrm{P}<0.05)$. (F) Representative photographs of immunohistochemical analysis of the Ki-67 antigen in tumors from SGC-7901-SH/NC groups; comparison of proliferation index is shown on the right $(* P<0.05)$.

To elucidate the potential functions of ZHX1 in GC cell lines, we established a stable ZHX1 upregulated NCI-N87 cell clone and a ZHX1 downregulated SGC-7901 cell clone. Cell growth was inhibited in cells upregulated for ZHX1 expression, as measured by CCK-8 assay, soft agar colony formation, and the nude mouse xenograft model. Reduction in endogenous ZHX1 level in SGC-7901 cells enhanced the cell growth in vitro and in vivo. Several studies have demonstrated that ZHX1 may play a pivotal role in cell growth and differentiation. Wang et al. [11] found that ectopic expression of ZHX1 in HCC cell lines resulted in inhibition of cell proliferation. In the current study, our results revealed that ZHX1 inhibits GC cell proliferation in vitro and in vivo. In addition, we found that ZHX1 could impair the migratory capacity of GC cell lines, which implicates that loss of ZHX1 expression in GC may facilitate metastasis.

Abnormal regulation of cell-cycle processes is a fundamental aspect of cancer cells [18]. The G1/S transition is essential for cell proliferation and its dysfunction could contribute to the carcinogenesis of many human cancers [19]. ZHX1 can interact with the activation domain of the A subunit of nuclear factor $Y$ (NF-YA). NF-Y is a trimeric transcriptional factor that comprises NF-YA, NF-YB, and NF-YC, and plays an important role in cell-cycle progression [20, 21]. ZHX2, another member of the ZHX family, can induce cell-cycle arrest by repressing expression of cyclins A and E [7]. However, the detailed molecular mecha- 
nism underlying how ZHX1 regulates the cell cycle is not clear, and more investigations should be performed. We observed that overexpression of ZHX1 can block the cell cycle at G0/G1 phase by decreasing the expression of cyclins D1 and E, and reduction of ZHX1 has the opposite effect. Moreover, we found that ZHX1 could induce cell apoptosis, which was associated with downregulation of anti-apoptotic protein Bcl2 and upregulation of apoptotic protein Bax and cleaved caspase-3. Apoptosis is a programmed cell death precisely regulated in normal cells. The imbalance of apoptosis and cell proliferation is a crucial event in tumor pathogenesis. Cancer cells can suppress apoptosis through a variety of molecular mechanisms, promoting the tumor progression [22]. Taken together, our findings suggest that ZHX1 plays a tumor suppressive role in GC cell lines and inhibits cell growth through inducing apoptosis and cell-cycle G1/S arrest.

In summary, we demonstrate, for the first time, that the expression of nuclear ZHX1 is downregulated in GC tissues and that ZHX1 can inhibit cell growth through inducing cell-cycle G1/S arrest and apoptosis. Thus, ZHX1 may functions as a tumor suppressor in GC. Further studies are warranted to clarify the detail of the molecular mechanism underlying these biological phenomena.

\section{Abbreviations}

GC: gastric cancer; ZHX1: zinc-fingers and homeoboxes 1; HCC: hepatocellular carcinoma; NLS: nuclear localization signal.

\section{Acknowledgments}

We thank Prof. Kazuya Yamada (Matsumoto University Graduate School of Health Science) for kindly providing the human pEGFP-ZHX1 plasmid. The project was supported by the Shanghai Municipal Education Commission (No. 13YZ025).

\section{Competing Interests}

The authors have declared that no competing interest exists.

\section{References}

1. Torre LA, Bray F, Siegel RL, Ferlay J, Lortet-Tieulent J, Jemal A. Global cancer statistics, 2012. CA: a cancer journal for clinicians. 2015; 65: 87-108.

2. Moon YW, Jeung HC, Rha SY, Yoo NC, Roh JK, Noh SH, et al. Changing patterns of prognosticators during 15-year follow-up of advanced gastric cancer after radical gastrectomy and adjuvant chemotherapy: a 15-year follow-up study at a single korean institute. Annals of surgical oncology. 2007; 14: 2730-7.

3. Barthelemy I, Carramolino L, Gutierrez J, Barbero JL, Marquez G, Zaballos A. zhx-1: a novel mouse homeodomain protein containing two zinc-fingers and five homeodomains. Biochemical and biophysical research communications. 1996; 224: 870-6.

4. Kawata H, Yamada K, Shou Z, Mizutani T, Yazawa T, Yoshino M, et al. Zinc-fingers and homeoboxes (ZHX) 2, a novel member of the ZHX family, functions as a transcriptional repressor. The Biochemical journal. 2003; 373: 747-57.
5. Yamada K, Kawata H, Shou Z, Hirano S, Mizutani T, Yazawa T, et al. Analysis of zinc-fingers and homeoboxes (ZHX)-1-interacting proteins: molecular cloning and characterization of a member of the ZHX family, ZHX3. The Biochemical journal. 2003; 373: 167-78.

6. Lv Z, Zhang M, Bi J, Xu F, Hu S, Wen J. Promoter hypermethylation of a novel gene, ZHX2, in hepatocellular carcinoma. American journal of clinical pathology. 2006; 125: 740-6.

7. Yue X, Zhang Z, Liang X, Gao L, Zhang X, Zhao D, et al. Zinc fingers and homeoboxes 2 inhibits hepatocellular carcinoma cell proliferation and represses expression of Cyclins A and E. Gastroenterology. 2012; 142: 1559-70.e2.

8. Nagel S, Schneider B, Meyer C, Kaufmann M, Drexler HG, Macleod RA. Transcriptional deregulation of homeobox gene ZHX2 in Hodgkin lymphoma. Leukemia research. 2012; 36: 646-55.

9. Nagel S, Schneider B, Rosenwald A, Meyer C, Kaufmann M, Drexler HG, et al. $\mathrm{t}(4 ; 8)(\mathrm{q} 27 ; \mathrm{q} 24)$ in Hodgkin lymphoma cells targets phosphodiesterase PDE5A and homeobox gene ZHX2. Genes, chromosomes \& cancer. 2011; 50: 996-1009.

10. Armellini A, Sarasquete ME, Garcia-Sanz R, Chillon MC, Balanzategui A, Alcoceba M, et al. Low expression of ZHX2, but not RCBTB2 or RAN, is associated with poor outcome in multiple myeloma. British journal of haematology. 2008; 141: 212-5.

11. Wang J, Liu D, Liang X, Gao L, Yue X, Yang Y, et al. Construction of a recombinant eukaryotic human ZHX1 gene expression plasmid and the role of ZHX1 in hepatocellular carcinoma. Molecular medicine reports. 2013; 8: 1531-6.

12. Yamada K, Kawata H, Matsuura K, Shou Z, Hirano S, Mizutani T, et al. Functional analysis and the molecular dissection of zinc-fingers and homeoboxes 1 (ZHX1). Biochemical and biophysical research communications. 2002; 297: 368-74

13. Yamada K, Printz RL, Osawa H, Granner DK. Human ZHX1: cloning, chromosomal location, and interaction with transcription factor NF-Y. Biochemical and biophysical research communications. 1999; 261: 614-21.

14. Ogata-Kawata H, Yamada K, Uesaka-Yoshino M, Kagawa N, Miyamoto K. BS69, a corepressor interacting with ZHX1, is a bifunctional transcription factor. Frontiers in bioscience : a journal and virtual library. 2007; 12: 1911-26.

15. Kim SH, Park J, Choi MC, Kim HP, Park JH, Jung Y, et al. Zinc-fingers and homeoboxes 1 (ZHX1) binds DNA methyltransferase (DNMT) 3B to enhance DNMT3B-mediated transcriptional repression. Biochemical and biophysical research communications. 2007; 355: 318-23.

16. Wang Z, Ma X, Cai Q, Wang X, Yu B, Cai Q, et al. MiR-199a-3p promotes gastric cancer progression by targeting ZHX1. FEBS letters. 2014; 588: 4504-12.

17. Zang $\mathrm{M}$, Zhang B, Zhang $\mathrm{Y}$, Li J, Su L, Zhu Z, et al. CEACAM6 promotes gastric cancer invasion and metastasis by inducing epithelial-mesenchymal transition via PI3K/AKT signaling pathway. PLoS One. 2014; 9: e112908.

18. Hanahan D, Weinberg RA. Hallmarks of cancer: the next generation. Cell. 2011; 144: 646-74.

19. Stein GS, van Wijnen AJ, Stein JL, Lian JB, Montecino M, Zaidi SK, et al. An architectural perspective of cell-cycle control at the G1/S phase cell-cycle transition. Journal of cellular physiology. 2006; 209: 706-10.

20. Chae HD, Kim J, Shin DY. NF-Y binds to both G1- and G2-specific cyclin promoters; a possible role in linking CDK2/Cyclin A to CDK1/Cyclin B. BMB reports. 2011; 44: 553-7.

21. Mantovani R. The molecular biology of the CCAAT-binding factor NF-Y. Gene. 1999; 239: 15-27.

22. Hassan M, Watari H, AbuAlmaaty A, Ohba Y, Sakuragi N. Apoptosis and molecular targeting therapy in cancer. BioMed research international. 2014; 2014: 150845. 